\title{
Interpretations and comments for expert consensus on the diagnosis and treatment of heat stroke in China
}

\author{
Shu-Yuan Liu ${ }^{1,2+}$, Qian Wang ${ }^{3+}$, Yun-Peng Lou ${ }^{4}$, Yan Gao ${ }^{5}$, Bo Ning ${ }^{6}$, Qing Song ${ }^{1 *}$ (D) and Hai-Ling Li ${ }^{4^{*}}$
}

\begin{abstract}
Heat stroke is the most severe type of heat illness, it is often accompanied by severe multiorgan damage and has a high fatality rate. In January 2020, based on new research evidence and the experiences of Chinese experts in heat stroke, the Expert Group of Heat Stroke Prevention and Treatment of the Chinese People's Liberation Army (PLA) and the Professional Committee of Critical Care Medicine of the Chinese PLA jointly issued a new Expert Consensus on the Diagnosis and Treatment of Heat Stroke in China. This article aims to interpret and supplement the major updates to the new consensus.
\end{abstract}

Keywords: Heat stroke, Definition, Diagnosis, Treatment, Targeted temperature management

Heat-related injury can cause a series of mild to severe pathophysiological changes, collectively referred to as heat illness [1]. Heat stroke, often accompanied by severe multiorgan damage, is the most severe type of heat illness and has high fatality rate. In January 2020, based on new research evidence and the experiences of Chinese experts in heat stroke, the Expert Group of Heat Stroke Prevention and Treatment of the Chinese PLA and the Professional Committee of Critical Care Medicine of the Chinese PLA jointly issued a new Expert Consensus on the Diagnosis and Treatment of Heat Stroke in China [2] (hereinafter referred to as the "new consensus"), in which the definition of heat stroke and diagnostic criteria were updated.

To date, there is no perfect definition of heat stroke. The most commonly used definition worldwide was Bouchama's definition that was proposed in 2002 [3];

\footnotetext{
* Correspondence: songqing3010301@sina.com; lihailing608@163.com †Shu-Yuan Liu and Qian Wang contributed equally to this work. ${ }^{1}$ Medical School of Chinese PLA, No. 28 of Fuxing Road, Beijing 100853, China

${ }^{4}$ Department of Intensive Care Unit, No. 971 Hospital of the People's Liberation Army Navy, No. 22 Minjiang Road, Qingdao 266071, China Full list of author information is available at the end of the article
}

this old definition served as a basis for the new definition of heat stroke posited in the new consensus. Although the onset of heat stroke is often associated with environmental factors (such as high temperature), this factor is not actually necessary. Some individuals may also develop heat stroke while exercising vigorously in an environment that is not too hot. In fact, the main mechanism of heat stroke is the imbalance between body heat production and dissipation, resulting in a large amount of heat accumulation in the body beyond endurance capacity, which causes extensive damage. A pathophysiological description added to the new definition makes it more consistent with the nature of heat stroke (see attachment 1 for details).

Contrary to the definition, the diagnostic criteria for heat stroke have rarely been clearly described in previous guidelines. Hyperthermia is the most prominent characteristic of heat stroke, and the core temperature is often used to distinguish the severity. However, in clinical practice, it was often found that some patients had significant manifestations of heat stroke when their measured temperature did not reach $40^{\circ} \mathrm{C}$ [4-6]. In consideration of this situation, the Japanese Association 
for Acute Medicine (JAAM) proposed new diagnostic criteria (modified JAAM criteria) in 2016 [4], in which body temperature was not included in the diagnostic criteria. In addition, not all individuals with a body temperature of more than $40^{\circ} \mathrm{C}$ experience heat stroke. Based on these considerations and the current situation of treatment in China, the new consensus proposed new diagnostic criteria consisting of two aspects, "medical history information" and "clinical manifestations". Heat stroke should be considered if the patient meets any of the medical history information criteria while also having any of the clinical presentations (if the symptoms cannot be explained by other reasons). The new consensus no longer treats core temperature as a necessary condition for clinical diagnosis, thus avoiding delays in treatment due to "diagnostic" problems (see attachment 2 for details).

To further standardize and emphasize cooling treatment, the new consensus introduced the concept of targeted temperature management (TTM). The accurate management of body temperature might be particularly important for heat stroke. It is important to choose the most effective way to control the body temperature according to the condition of the scene. It should be emphasized that any cooling method or combination of two or more technologies should be used until more effective cooling measures are achieved. However, there is still no strong evidence to determine the optimal target temperature for cooling therapy. Most studies recommend a cooling endpoint between $38.0^{\circ} \mathrm{C}$ and $39.0^{\circ} \mathrm{C}$. It should be noted that these recommendations were based only on the results observed in the field treatment of athletes and lacked strong scientific evidence. In theory, mild hypothermia does not occur until the core temperature drops to $35.0^{\circ} \mathrm{C}$, so systemic cooling in patients with heat stroke can be continued until clinical improvement or rectal temperature ranges from $37.0^{\circ} \mathrm{C}$ to $38.0^{\circ} \mathrm{C}$ as opposed to depending on a certain temperature node. Combined with the above evidence and debates, the new consensus suggests that the goal of core temperature management is to maintain the rectal temperature at $37.0^{\circ} \mathrm{C}$ to $38.5^{\circ} \mathrm{C}$, which is a broad range (see attachment 3 for details).

\section{Supplementary information}

Supplementary information accompanies this paper at https://doi.org/10. 1186/s40779-020-00266-4.

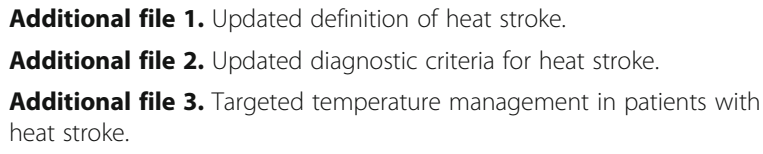

\section{Abbreviations}

JAAM: Japanese Association for Acute Medicine; TTM: Targeted temperature management

\section{Acknowledgments}

Many thanks to the Expert Group of Heat Stroke Prevention and Treatment of the Chinese PLA for their valuable suggestions.

\section{Authors' contributions}

SYL, QW and YPL carried out information retrieval and wrote a draft of the paper. QS, BN, YG, HLL and QW gave specific instructions for writing as the members of the Expert Group of Heat Stroke Prevention and Treatment of Chinese PLA. QS and HLL reviewed the draft paper. All authors read and approved the final manuscript.

\section{Funding}

This study was supported by the military medical innovation project fund (18CXZ019, 18CXZ024), a Chinese military fund dedicated to the prevention and control of heat-related illness with no commercial interest.

\section{Availability of data and materials}

The datasets used and/or analyzed are available from the corresponding authors on reasonable request.

Ethics approval and consent to participate

Not applicable.

Consent for publication

Not applicable.

\section{Competing interests}

No conflicts of interest to disclose.

\section{Author details}

${ }^{1}$ Medical School of Chinese PLA, No. 28 of Fuxing Road, Beijing 100853, China. ${ }^{2}$ Emergency Department, Sixth Medical Center, Chinese PLA General Hospital, No. 6 of Fucheng Road, Beijing 100048, China. ${ }^{3}$ Emergency Department, Third Medical Center, Chinese PLA General Hospital, No. 69 of Yongding Road, Beijing 100039, China. ${ }^{4}$ Department of Intensive Care Unit, No. 971 Hospital of the People's Liberation Army Navy, No. 22 Minjiang Road, Qingdao 266071, China. ${ }^{5}$ Emergency Department, General Hospital of Northern Theater Command of Chinese PLA, No. 83 of Wenhua Road, Shenyang 110016, China. ${ }^{6}$ Department of Intensive Care Unit, Air Force Medical Center of China, No.30 of Fucheng Road, Beijing 100142, China.

Received: 1 April 2020 Accepted: 29 July 2020

Published online: 06 August 2020

\section{References}

1. Armed Forces Health Surveillance Branch. Update: Heat illness, active component, U.S. Armed forces, 2017. MSMR. 2018;25(4):6-12.

2. Liu SY, Song JC, Mao HD, Zhao JB, Song Q, et al. Expert consensus on the diagnosis and treatment of heat stroke in China. Mil Med Res. 2020;7:1.

3. Bouchama A, Knochel JP. Heat stroke. N Engl J Med. 2002;346(25):1978-88.

4. Hifumi T, Kondo Y, Shimazaki J, Oda Y, Shiraishi S, Wakasugi M, et al. Prognostic significance of disseminated intravascular coagulation in patients with heat stroke in a nationwide registry. J Crit Care. 2018;44:306-11.

5. Knoll JM, Knight LR, Quiroz D, Popat SM, Pederson TG, Morton-Gonzaba N. Variation in clinical presentations and outcomes of heat stroke victims in the mass-casualty setting. J Emerg Med. 2019;57(6):866-70.

6. Cha S, Kwon BS, Hong N, Park JS, Byun SK, Choi SC, et al. Ischemic colitis associated with Rhabdomyolysis and heat stroke after an intense exercise in young adult. Korean J Gastroenterol. 2019;74(2):115-8. 